PROCEEDINGS OF THE

AMERICAN MATHEMATICAL SOCIETY

Volume 140, Number 2, February 2012, Pages 701-705

S 0002-9939(2011)10929-8

Article electronically published on June 21, 2011

\title{
ON THE BOUNDARY OF KÄHLER CONES
}

\author{
XIANGWEN ZHANG
}

(Communicated by Jianguo Cao)

\begin{abstract}
We study some geometric properties of a compact Kähler manifold $\left(M^{n}, g\right)$ under a certain condition on the bisectional curvature. As an application, we give a new proof for an earlier result which asserts that any boundary class of the Kähler cone of $M^{n}$ can be represented by a $C^{\infty}$ closed $(1,1)$ form that is parallel and everywhere nonnegative.
\end{abstract}

Let $\left(M^{n}, g\right)$ be a compact Kähler manifold. Denote by

$$
H(M)=H_{\mathbb{C}}^{1,1}(M)=H^{1,1}(M) \cap H^{2}(M, \mathbb{C})
$$

the vector space of real $(1,1)$ classes. Write $\mathcal{K}(M)$ for the Kähler cone in $H(M)$, namely, the convex cone formed by all the cohomology classes that can be represented by smooth closed $(1,1)$ forms that are everywhere positives. We are interested in the boundary set $\mathcal{B}=\overline{\mathcal{K}} \backslash \mathcal{K}$ of the Kähler cone. We will call a (nontrivial) cohomology class $\alpha$ in $\mathcal{B}$ a boundary Kähler class of $M$. It would be an interesting question to ask when $\alpha$ can be represented by a closed, smooth $(1,1)$ form that is everywhere nonnegative. The existence of such forms often has direct geometric applications in the study of Monge-Ampère foliations or Kähler submersions.

Recently, this problem has been studied by D. Wu, S.T. Yau and F. Zheng 5 . They interpreted the geometric problem by a degenerate complex Monge-Ampère equation and proved the existence of a smooth solution to the equation through a delicate way. The main result in their paper is as follows:

Theorem (Wu - Yau - Zheng). Let $\left(M^{n}, g\right)$ be a compact manifold satisfying the following curvature condition: for any orthogonal tangent frame $e_{1}, \cdots, e_{n}$ at any $x \in M$, and for any real numbers $a_{1}, \cdots, a_{n}$ :

$$
\sum_{i, j=1}^{n} R_{i \bar{i} j \bar{j}}\left(a_{i}-a_{j}\right)^{2} \geq 0
$$

Then any boundary class of the Kähler cone of $M^{n}$ can be represented by a $C^{\infty}$ closed $(1,1)$ form that is everywhere nonnegative.

As pointed out in [5], when $n=2$, the curvature condition $(*)$ simply implies $R_{u \bar{u} v \bar{v}} \geq 0$ for any pair of orthogonal tangent vectors $\mathrm{u}, \mathrm{v}$ of type $(1,0)$, which means that $M^{2}$ has nonnegative orthogonal bisectional curvature. For $n>2$, it's easy to give an example and see that the condition $(*)$ becomes much less restrictive than nonnegative orthogonal bisectional curvature. So, it should be interesting to

Received by the editors October 24, 2010 and, in revised form, December 1, 2010.

2010 Mathematics Subject Classification. Primary 53B35, 51M99.

(C)2011 American Mathematical Society 
study some geometric properties of the compact manifolds under this curvature condition.

In 1981, A. Howard, B. Smyth and H. Wu [6] proved that

Lemma (Howard - Smyth - Wu). If a compact Kähler manifold $M$ satisfies curvature condition $(*)$, then all harmonic forms of type $(1,1)$ are parallel. In particular, the Hodge number $h^{1,1}$ is equal to 1 if $M$ is locally irreducible.

Inspired by this nice result, we can prove the following geometric property for the manifolds with curvature condition $(*)$.

Theorem 1. Let $\left(M^{n}, g\right)$ be a compact Kähler manifold satisfying the curvature condition $(*)$. Then, for any closed $(1,1)$ form $\Phi$ on $\left(M^{n}, g\right)$, we can find $\tilde{\Phi} \in[\Phi]$, such that $\tilde{\Phi}$ is parallel. In particular, for any closed $(1,1)$ form $\alpha$, we have

$$
[\alpha]=\left[\beta+\lambda_{s} \omega_{0}\right],
$$

where $\beta$ is a nonnegative $C^{\infty}$ closed $(1,1)$ form on the boundary of the Kähler cone, $\lambda_{s}$ is a constant depending on $\beta$, and $\omega_{0}$ is the Kähler form on $\left(M^{n}, g\right)$.

Before giving the proof for our main theorem, we state a fundamental lemma which can be found in [1].

Lemma (1] (2.33)). Let $\phi$ be a closed $(1,1)$ form on a compact Kähler manifold. Then $\phi$ is harmonic if and only if its trace is constant.

Proof of Theorem [1. Consider the equation

$$
\sigma_{1}\left(\omega_{0}+\Phi+\partial \bar{\partial} v\right)=C
$$

where $C$ is some constant to be determined. The above equation is equivalent to

$$
\triangle v=C-\left(n+\operatorname{Tr}_{g} \Phi\right) .
$$

By the standard theory of partial differential equations, we know that $\triangle u=f$ is solvable if and only if $\int_{M} f=0$. So, if we choose

$$
C=n+\frac{1}{\operatorname{Vol}(M)} \int_{M} \omega_{0}^{n-1} \wedge \Phi,
$$

there is a smooth solution of the equation (0.1).

Let $\tilde{\Phi}=\Phi+\partial \bar{\partial} v$. Then the equation is

$$
\sigma_{1}\left(\omega_{0}+\tilde{\Phi}\right)=C
$$

By the previous two lemmas, we can assert that $\tilde{\Phi}$ is a smooth parallel $(1,1)$ form and $\tilde{\Phi} \in[\Phi]$.

Let $\lambda_{s}$ be the smallest eigenvalue of the $(1,1)$ form $\tilde{\Phi}$ (under the fixed orthonormal frame) and define

$$
\phi=\tilde{\Phi}-\lambda_{s} \omega_{0}
$$

Then it is not hard to see that $\phi$ is nonnegative everywhere on $M^{n}$ and on the boundary of the Kähler cone.

Thus, for any closed $(1,1)$ form $\alpha$, we can find a nonnegative closed $(1,1)$ form $\beta$ on the boundary of the Kähler cone such that

$$
[\alpha]=\left[\beta+\lambda_{s} \omega_{0}\right],
$$

where $\omega_{0}$ is the Kähler form. 
As an application of the above theorem, we give a new proof for the main theorem in [5].

Corollary 1. Let $\left(M^{n}, g\right)$ be a compact manifold satisfying the curvature condition (*). Then any boundary class of the Kähler cone of $M^{n}$ can be represented by a $C^{\infty}$ closed $(1,1)$ form that is parallel and everywhere nonnegative.

Proof of Corollary 1. Suppose $\alpha$ is a closed $(1,1)$ form on the boundary of the Kähler cone. By the definition of boundary Kähler class, we know that there exists a sequence of smooth closed $(1,1)$ forms $\left\{\omega_{m}\right\}$ which are everywhere positive and $\omega_{m} \longrightarrow \alpha$ as $m \longrightarrow \infty$.

Now, consider the integration to be a continuous functional on the form space $h^{1,1}(M)$, and by the convergence, we get

$$
\int_{M} \omega_{m}^{k} \wedge \omega_{0}^{n-k} \longrightarrow \int_{M} \alpha^{k} \wedge \omega_{0}^{n-k}, \quad k=0,1, \cdots, n .
$$

So, $\int_{M} \alpha^{k} \wedge \omega_{0}^{n-k} \geq 0$ for any $k=0,1, \cdots, n$.

According to the proof of Theorem 1, there is a parallel closed $(1,1)$ form $\tilde{\alpha} \in[\alpha]$. Thus, the eigenvalues of $\tilde{\alpha}$ are all constant on $M^{n}$ and

$$
\begin{aligned}
& 0 \leq \int_{M} \alpha^{k} \wedge \omega_{0}^{n-k}=\int_{M} \tilde{\alpha}^{k} \wedge \omega_{0}^{n-k}=\sigma_{k}(\tilde{\alpha}) \int_{M} \omega_{0}^{n} \\
\Longrightarrow \quad & \sigma_{k}(\tilde{\alpha}) \geq 0, \quad k=0,1, \cdots, n,
\end{aligned}
$$

which means that $\tilde{\alpha}$ is in the $\Gamma_{n}$ convex cone. So, $\tilde{\alpha} \in[\alpha]$ is nonnegative everywhere.

Remark 1. Let $(M, g)$ be a compact Kähler manifold and let $\omega$ be the Kähler form of $g$. For any real $(1,1)$ form $\alpha$, we can define the $k$-th symmetric function for $\alpha$ with respect to $\omega$ as

$$
\alpha^{k} \wedge \omega^{n-k}=\sigma_{k}(\alpha) \omega^{n} .
$$

Denote the space of real $(1,1)$ forms by $\Lambda_{\mathbb{R}}^{1,1}$. We define the $k$-convex cone by

$$
\Gamma_{k}=\left\{\alpha \in \Lambda_{\mathbb{R}}^{1,1} \mid \sigma_{j}(\alpha) \geq 0, j=1, \cdots, k\right\} .
$$

To study the Betti number for a compact connected Kähler manifold under some curvature conditions is also a very interesting topic in geometry. In 1965, Bishop and Goldberg [2] showed that any compact Kähler manifold $M^{n}$ with positive bisectional curvature must have its second Betti number equal to 1. Later, Goldberg and Kobayashi [4] introduced the conception of holomorphic bisectional curvature and proved that the second Betti number of a compact connected Kähler manifold $M$ with positive holomorphic bisectional curvature is one. Now, if we restrict the curvature condition to be the following so-called quasi $-(*)$, we can also get the similar geometric property.

Definition 1. We say that a compact manifold $\left(M_{n}, g\right)$ satisfies the quasi- $(*)$ curvature condition if it satisfies: for any orthogonal tangent frame $e_{1}, \cdots, e_{n}$ at any $x \in M$, and for any real numbers $a_{1}, \cdots, a_{n}, \sum_{i, j=1}^{n} R_{i \bar{i} j \bar{j}}\left(a_{i}-a_{j}\right)^{2} \geq 0$ holds everywhere and is strictly positive at least at one point.

Theorem 2. Let $\left(M_{n}, g\right)$ be a compact Kähler manifold satisfying the quasi-(*) curvature condition. Then $\operatorname{dim} h^{1,1}(M, \mathbb{R})=1$. 
Proof. The proof follows the well-known technique due to Bochner and Lichnerowicz.

Let $\xi$ be a closed $(1,1)$ form on a compact Kähler manifold $M^{n}$. For any fixed point on $M^{n}$, we can choose local coordinates $\left\{z_{1}, \cdots, z_{n}\right\}$ such that

$$
g_{\alpha \bar{\beta}}=\delta_{\alpha \beta}, \frac{\partial g_{\alpha \bar{\beta}}}{\partial z_{i}}=\frac{\partial g_{\alpha \bar{\beta}}}{\partial z_{\bar{i}}}=0 \text { and } \xi=\xi_{i \bar{j}} d z^{i} \wedge d z^{\bar{j}} .
$$

We consider the function $\phi=\sigma_{2}\left(g^{i \bar{l}} \xi_{l \bar{j}}\right)$ and denote $W=\left(w_{i \bar{j}}\right)=\left(g^{i \bar{l}} \xi_{l \bar{j}}\right)$. Then we have

$$
\begin{aligned}
\phi_{\alpha} & =\sigma_{1}(W \mid i)\left(g^{i \bar{l}} \xi_{l \bar{i}}\right)_{\alpha}, \\
\phi_{\alpha \bar{\beta}} & =\sigma_{1}(W \mid i)\left(g^{i \bar{l}} \xi_{\bar{l}}\right)_{\alpha \bar{\beta}}+\left(g^{i \bar{l}} \xi_{\bar{l} \bar{i}}\right)_{\alpha}\left(g^{i \bar{k}} \xi_{k \bar{i}}\right)_{\bar{\beta}}-\left(g^{i \bar{l}} \xi_{\bar{l}}\right)_{\alpha}\left(g^{i \bar{k}} \xi_{k \bar{i}}\right)_{\bar{\beta}} .
\end{aligned}
$$

By the proof of Theorem 1 we know there is a closed $(1,1)$ form (which we still denote by $\xi)$ in $[\xi]$ such that

$$
F(\xi)=\sigma_{1}\left(g^{i \bar{l}} \xi_{l \bar{j}}\right)=C,
$$

where $C$ is some constant.

By (0.6), we have

$$
\begin{aligned}
F^{\alpha \bar{\beta}} & =g^{\alpha \bar{\beta}}=\delta_{\alpha \beta}, \\
\sigma_{1}\left(g^{\alpha \bar{\eta}} \xi_{\eta \bar{\beta}}\right)=C & \Longrightarrow \sum_{i}^{n} \xi_{i \bar{i}, \alpha}=0 \\
\sigma_{1}\left(g^{\alpha \bar{\eta}} \xi_{\eta \bar{\beta}}\right)=C & \Longrightarrow g_{, i \bar{\eta}}^{\alpha \bar{\eta}} \xi_{\eta \bar{\beta}}+g^{\alpha \bar{\eta}} \xi_{\eta \bar{\beta}, i \bar{i}}=0 \\
& \Longrightarrow \xi_{\alpha \bar{\alpha}, i \bar{i}}=-g_{, i \bar{i}}^{\alpha \bar{\eta}} \xi_{\eta \bar{\alpha}}=-g_{, i \bar{\alpha}}^{\alpha \bar{\alpha}} \xi_{\alpha \bar{\alpha}} .
\end{aligned}
$$

Thus, by direct computation, we can get that

$$
\begin{aligned}
(0.9) F^{\alpha \bar{\beta}} \phi_{\alpha \bar{\beta}} & =\sigma_{1}(W \mid i)\left(g^{i \bar{l}} \xi_{l \bar{i}}\right)_{\alpha \bar{\alpha}}+\left(g^{i \bar{l}} \xi_{l \bar{i}}\right)_{\alpha}\left(g^{i \bar{k}} \xi_{k \bar{i}}\right)_{\bar{\alpha}}-\left(g^{i \bar{l}} \xi_{l \bar{i}}\right)_{\alpha}\left(g^{i \bar{k}} \xi_{k \bar{i}}\right)_{\bar{\alpha}} \\
& =\sigma_{1}(W \mid i)\left(g_{, \alpha \bar{\alpha}}^{i \bar{i}} \xi_{i \bar{i}}+\xi_{i \bar{i}, \alpha \bar{\alpha}}\right)+\xi_{i \bar{i}, \alpha} \xi_{j \bar{j}, \bar{\alpha}}-\xi_{i \bar{j}, a} \xi_{i \bar{j}, \bar{\alpha}} \\
& =\sigma_{1}(W \mid i)\left(g_{, \alpha \bar{\alpha}}^{i \bar{i}} \xi_{i \bar{i}}+\xi_{\alpha \bar{\alpha}, i \bar{i}}\right)+\xi_{i \bar{i}, \alpha} \xi_{j \bar{j}, \bar{\alpha}}-\xi_{i \bar{j}, a} \xi_{i \bar{j}, \bar{\alpha}} \\
& =\sigma_{1}(W \mid i)\left(g_{, \alpha \bar{\alpha}}^{i \bar{i}} \xi_{i \bar{i}}-g_{, i \bar{\alpha}}^{\alpha \bar{\alpha}} \xi_{\alpha \bar{\alpha}}\right)-\sum_{i, j}\left|\nabla \xi_{i \bar{j}}\right|^{2}+\left(\sum_{i=1}^{n} \xi_{i \bar{i}, \alpha}\right)^{2} \\
& =\sigma_{1}(W \mid i)\left(-R_{i \bar{i} \alpha \bar{\alpha}} \xi_{i \bar{i}}+R_{i \bar{i} \alpha \bar{\alpha}} \xi_{\alpha \bar{\alpha}}\right)-\sum_{i, j}\left|\nabla \xi_{i \bar{j}}\right|^{2} \\
& =-\frac{1}{2} \sum_{i \alpha} R_{i \bar{i} \alpha \bar{\alpha}}\left(\sigma_{1}(W \mid i)-\sigma_{1}(W \mid \alpha)\right)\left(\xi_{i \bar{i}}-\xi_{\alpha \bar{\alpha}}\right)-\sum_{i, j}\left|\nabla \xi_{i \bar{j}}\right|^{2} \\
& =-\frac{1}{2} \sum_{i \alpha} R_{i \bar{i} \bar{\alpha} \bar{\alpha}}\left(\xi_{i \bar{i}}-\xi_{\alpha \bar{\alpha}}\right)^{2}-\sum_{i, j}\left|\nabla \xi_{i \bar{j}}\right|^{2} .
\end{aligned}
$$

From the second to third equality, we used the fact that $\xi$ is a closed $(1,1)$ form, which gives us

$$
\xi_{\alpha \bar{\alpha}, i}=\xi_{i \bar{\alpha}, \alpha}, \quad \xi_{\alpha \bar{\alpha}, \bar{i}}=\xi_{\alpha \bar{i}, \bar{\alpha}}
$$


From equality (0.9), we can see that if the compact Kähler manifold $M^{n}$ satisfies the curvature condition $(*)$, then

$$
F^{\alpha \bar{\beta}} \phi_{\alpha \bar{\beta}}=-\frac{1}{2} \sum_{i, \alpha} R_{i \bar{i} \alpha \bar{\alpha}}\left(\xi_{i \bar{i}}-\xi_{\alpha \bar{\alpha}}\right)^{2}-\sum_{i, j}\left|\nabla \xi_{i \bar{j}}\right|^{2} \leq 0 .
$$

Finally, by the strong maximal principle, we assert that $\phi \equiv$ constant. Thus,

$$
\sigma_{1}\left(g^{i \bar{l}} \xi_{l \bar{j}}\right)=\text { constant and } \sigma_{2}\left(g^{i \bar{l}} \xi_{l \bar{j}}\right)=\text { constant, }
$$

from which we can get that $\xi_{i \bar{i}}$ are constants on $M^{n}$. Furthermore, if the manifold satisfies the quasi- $(*)$ curvature condition, by (0.11) , we can see that $\xi_{i \bar{i}}=\xi_{\alpha \bar{\alpha}}$ for $i, \alpha=1, \cdots, n$. Thus, $\xi=\lambda \omega_{0}$, where $\lambda$ is a constant. So, $\operatorname{dim} h^{1,1}(M)=1$.

Remark 2. For the results in 2, 4, 6, the restriction of the bisectional curvature makes the Ricci tensor of $M$ to be positive. So there are no nontrivial holomorphic 2-forms on $M$ (cf. Bochner [8), i.e., $H^{2,0}(M)=H^{0,2}(M)=0$, i.e., $b_{2}(M)=$ $\operatorname{dim} H^{2}(M)=\operatorname{dim} h^{1,1}(M)$. But, in our case, we have no information about the positivity of the Ricci tensor from the quasi- $(*)$ curvature condition. Thus, we cannot get $b_{2}(M)=1$.

\section{ACKNOWLEDGEMENTS}

The author wishes to thank his advisor, Professor Pengfei Guan, for the helpful discussions on this problem and also for his continuous support and encouragement. The author would also like to thank the authors of [5] for bringing this question and also the curvature condition $(*)$ to our attention. Finally, we wish to thank the referee for valuable comments.

\section{REFERENCES}

[1] A. Besse, Einstein manifolds. Ergeb. Math. Grenzgeb. Band 10. Springer-Verlag, Berlin and New York, 2009. MR867684 (88f:53087)

[2] R.L. Bishop and S. Goldberg, On the second cohomology group of a Kähler manifold of positive curvature. Proc. Amer. Math. Soc. 16 (1965), 119-122. MR0172221 (30:2441)

[3] S. Bochner, Curvature and Betti numbers. Ann. of Math. (2) 49 (1948), 379-390. MR0025238 (9:618d)

[4] S. Goldberg and S. Kobayashi, Holomorphic bisectional curvature. J. Differential Geometry 1 (1967), 225-233. MR0227901(37:3485)

[5] D. Wu, S-T. Yau and F. Zheng, A degenerate Monge-Ampère equation and the boundary classes of Kähler cones. Math. Res. Lett. 16 (2009), no. 2, 365-374. MR2496750|(2010c:32061)

[6] A. Howard, B. Smyth and H. Wu, On compact Kähler manifolds of nonnegative bisectional curvature. I, Acta Math. 147 (1981), 51-56. MR631087 (83e:53064a)

[7] H. Wu, On compact Kähler manifolds of nonnegative bisectional curvature. II. Acta Math. 147 (1981), 57-70. MR631088 (83e:53064b)

[8] K. Yano and S. Bochner, Curvature and Betti numbers. Annals of Math. Studies. No. 32, Princeton University Press, Princeton, NJ, 1953. MR0062505 (15:989f)

Department of Mathematics and Statistics, McGill University, Montreal, Quebec, H3A 2K6, CANADA

E-mail address: xzhang@math.mcgill.ca 